\title{
INFRARED SPECTRA OF RECENT NOVAE
}

\author{
Yvette Andrillat \\ Laboratoire d'Astronomie, USTL, 34060 Montpellier Cedex 1 \\ and URA 1281, OPM, France
}

Léo Houziaux

Université de Mons, Département d'Astrophysique

B 7000 Mons, Belgium

We present CCD spectra of novae PW Vul, QS And, Herculis 87 and V1819 Cyg obtained

in the wavelength range 680 to $1080 \mathrm{~mm}$ at the Cassegrain focus of the 193-cm telescope at the Observatoire de Haute-Provence (CNRS, France). The reciprocal dispersion is $23 \mathrm{~nm} / \mathrm{mm}$.

$\begin{array}{lcrccc}\text { Nova } & \text { Date } & \text { Day } & \begin{array}{c}\text { Table I } \\ \text { Comp. Star }\end{array} & \begin{array}{c}\text { Exp time } \\ (\text { sec })\end{array} & \text { E(B-V) } \\ \text { PW Vu1 } & \text { Sept 29'85 } & 424 & \text { o Peg } & 7200 & 0.45 \\ \text { QS And } & \text { Sept 9'87 } & 273 & \text { o Peg } & 8100 & 0.063 \\ \text { Her } 87 & \text { June 5 '87 } & 125 & 109 \text { Vir } & 5400 & 0.4 \\ \text { Her 87 } & \text { Sept 12'87 } & 224 & \text { o Peg } & 900 & 0.4 \\ \text { V1819 Cyg Sept 9' } 87 & 398 & \text { o Peg } & 8100 & 0.7\end{array}$

Table I gives for each nova the date of observation, the number of days elapsed since maximum light in the visible, the comparison star used, the exposure time in seconds and the $E(B-V)$ value adopted in view of their galactic Iocation. These spectra have been used for deriving the emission line fluxes given in table II.

Figures 1 to 5 display the spectra who refer to the nebular phase. The spectral range in the figures has been limited to the portions of the spectrum where the noise is tolerable. It should be noted that these tracings are generally the mean of several spectra taken at short time intervals. The mean fluxes are divided by the continuum in order to obtain a clear presentation of the various features. The ordinates should thus not be used for comparing line intensities. Identifications are reported on the figures where the wavelength scale is given in Angströms.

PW Vul (figures $I$ and 2). Spectra of this nova have been published namely by Kenyon and Wade (1986). The present spectra refer to day 424. The spectrum on day 90 is given for comparison (see Andrillat and Houziaux, 1987). Spectral changes are important between these two dates. On day 424, CI, NI, and SI 1ines have disappeared whereas Paschen lines are reinforced. HeII lines are strong. SIII lines are predominant and the coronal line SVIII at $991.8 \mathrm{~nm}$ is present. An unidentified feature at $833.5 \mathrm{~nm}$ is clearly seen on the red wing of the OI line.

QS Andromedae (figure 3). On our spectrum taken on day 273, Paschen lines are visible from P7 to P12. OI and HeI lines are present, as well as the HeII line at $1012.3 \mathrm{~nm}$ (not shown on fig 3 ). The nebular lines seen in the spectrum belong to OII, AIII, AIV, SIII, and SVIII. The unidentified feature at $833.5 \mathrm{~nm}$ is a narrow line relatively intense.

Nova Herculis 1987 (figures 4 and 5). We observed this nova at two dates during the nebular phase. Between days 125 and 224, spectra have strongly evolved. S III 1ines have become quite strong on day 224. S VIII, already visible on day 125 is still there on day 224. At that date, the spectrum exhibits the unidentified structure at $833.5 \mathrm{~nm}$; it is a narrow line located on the red wing of the OI+NI blend. The lines of several ions show a double structure.

V 1819 Cygni. Spectra of this nova have already ben published and described (Andrillat and Houziaux, 1989). We report in this paper the emission line fluxes for day 398.

The emission line fluxes have been obtained in comparing the novae flux distributions to that of 0 Peg and $109 \mathrm{Vir}$, whose monochromatic magnitudes are taken from Cochran 
Table II.Emission line fluxes (in units of $10^{-13} \mathrm{erg} \mathrm{cm}^{-2} \mathrm{~s}^{-1}$ )

$\begin{array}{cccccc}(\mathrm{nm}) \quad \text { Ion } & \text { PW Vu1 } & \text { QS And } & \text { Her } 87 & \text { Her } 87 & \text { V1819 Cyg } \\ & 424 & 273 & 125 & 224 & 398\end{array}$

\begin{tabular}{|c|c|c|c|c|c|c|}
\hline 667.8 & $\mathrm{He} \mathrm{I}$ & & 2.21 & & 0.234 & 11.2 \\
\hline 700.6 & $\mathrm{~A} \mathrm{~V}$ & & & & & 4.4 \\
\hline 706.5 & & & 1.47 & 5.76 & 0.288 & 2.6 \\
\hline 713.5 & A III & & 1.51 & 2.73 & 0.485 & 2.0 \\
\hline 723.5 & A IV & & 3.04 & 4.32 & 0.297 & 3.3 \\
\hline 733.0 & $0 \mathrm{II}$ & & 24.8 & 6.75 & 2.31 & 41.4 \\
\hline 753.4 & $\mathrm{He}$ II & & 0.39 & & 0.124 & 9.9 \\
\hline 772.5 & C IV (b1) & & 2.25 & 9.08 & 0.761 & \\
\hline 775.1 & A III & & & & & 6.7 \\
\hline 789.6 & $\mathrm{Fe} X I$ & 0.80 & & & & 1.4 \\
\hline 822.0 & $0 \mathrm{I}$ & & 1.96 & 4.61 & 0.414 & \\
\hline 822.4 & $\mathrm{He}$ II & 2.05 & & & & 5.3 \\
\hline 833.5 & $?$ & & 0.33 & & 0.09 & \\
\hline 844.6 & $0 \mathrm{I}$ & 0.50 & 0.73 & 6.10 & 0.117 & 4.4 \\
\hline 875.0 & $\mathrm{H}(\mathrm{P} 12)$ & 0.067 & & & 0.096 & 1.6 \\
\hline 886.8 & H (P11) & 0.08 & & 2.5 & & 1.1 \\
\hline 906.9 & $S$ III & 3.34 & & & 0.676 & \\
\hline 922.9 & H (P9) & 2.67 & & 7.18 & 0.62 & 4 \\
\hline 953.9 & P8+S III & 4.8 & & & 0.96 & \\
\hline 954.8 & & & & 5.4 & & 4.7 \\
\hline 991.8 & S VIII & 0.42 & & 3.84 & 0.14 & 1.9 \\
\hline
\end{tabular}

(1980, 1981). Differential atmospheric absorption between the novae and the comparison stars have been taken into account in using the mean atmospheric absorption curve given by Mianes (1963). The fluxes given in table II have been corrected for interstellar extinction in making use of the $E(B-V)$ values of table $I$ and Seaton's mean extinction curve. Because of noise, atmospheric absorption bands, and difficulties in setting the continuum level, the accuracy of these fluxes is around $\pm 25 \%$.

\section{REFERENCES}

Andrillat Y. and Houziaux L. 1987 Astron. Astrophys.Supp1.Ser. 67, 111

Andrillat Y. and Houziaux L. 1989 Mon. Not. R. astr. Soc. 238, 29P

Cochran A.L. 1980 Publications in Astron. $n^{\circ} 16$, University of Texas Cochran A.L. 1981 Astrophys. J. Supp1. Ser. 45, 83

Kenyon S.J. and Wade R.A. 1986 Pub1. Astron. Soc. Pacific, 98, 935

Mianes P. 1963 Ann. d'Astrophys. 26, 17

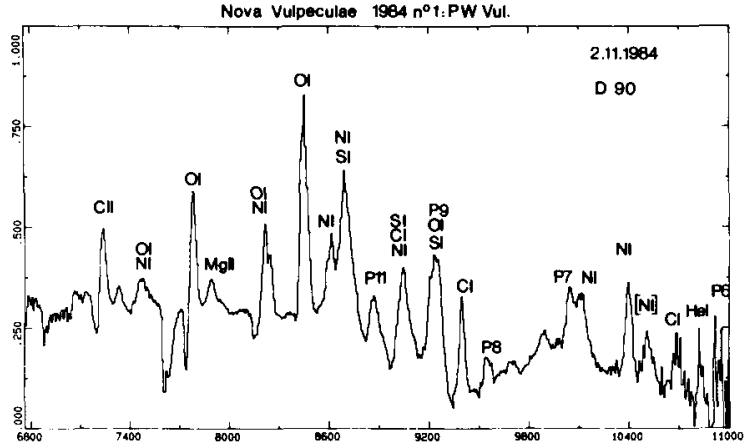

Figure 1

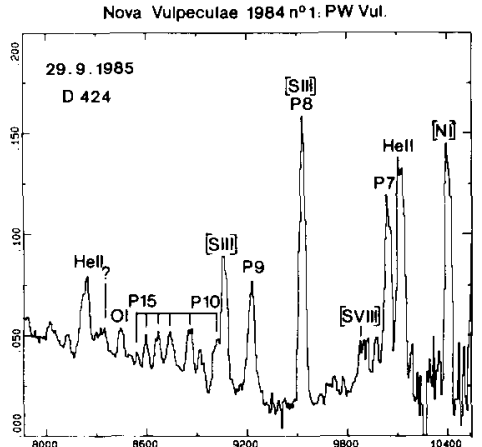

Figure 2 


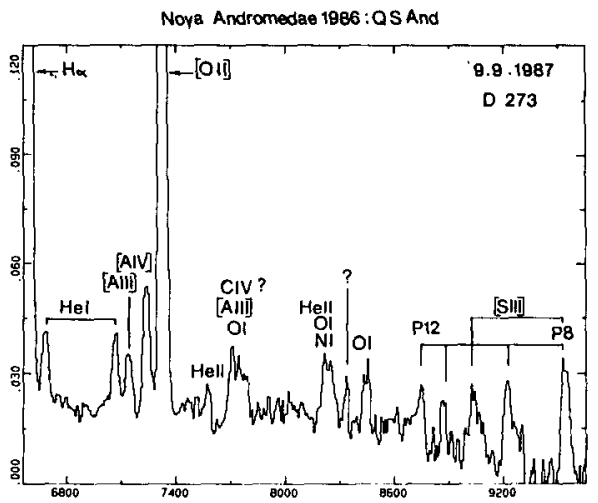

Figure 3

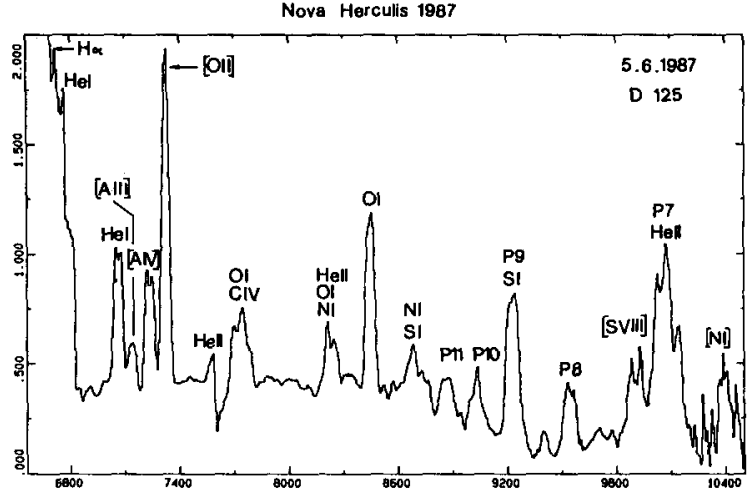

Figure 4

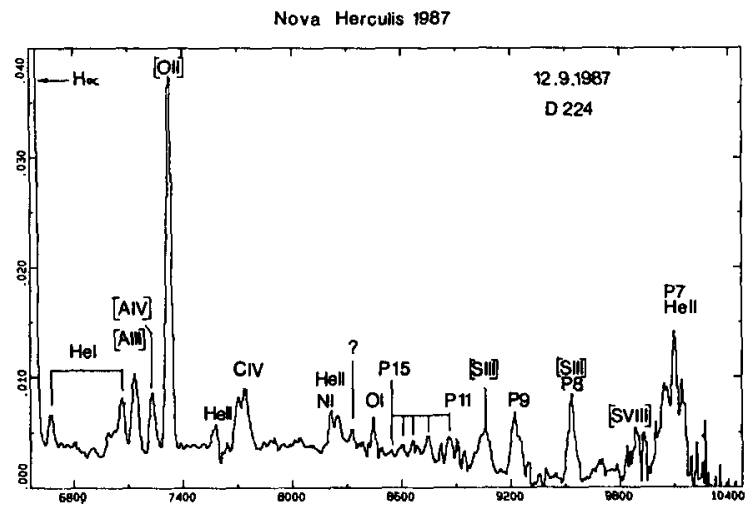

